\title{
Hadronic resonance production with ALICE at the LHC
}

\section{Sergey Kiselev* for the ALICE collaboration}

Institute for Theoretical and Experimental Physics, Moscow, Russia

E-mail: Sergey.Kiselev@cern.ch

The hadronic resonances $\mathrm{K}^{*}(892)^{0}$ and $\phi(1020)$ have been measured by the ALICE experiment at the LHC in $\mathrm{pp}, \mathrm{p}-\mathrm{Pb}$ and $\mathrm{Pb}-\mathrm{Pb}$ collisions. Transverse momentum spectra, yields, ratio to stable particles, nuclear modification factors and comparison with model predictions are discussed. The ALICE results are compared with data obtained at lower energies.

XXII International Baldin Seminar on High Energy Physics Problems,

15-20 September 2014

JINR, Dubna, Russia

\footnotetext{
* Speaker.
} 


\section{Introduction}

Resonance production plays an important role both in elementary and in heavy-ion collisions. In pp and $\mathrm{p}-\mathrm{A}$ collisions, it provides the baseline for heavy-ion collisions and it can be used to study system size dependence and the role of cold nuclear matter. In heavy-ion collisions, it contributes to the systematic study of in-medium parton energy loss and its flavor dependence. Moreover, effects related to the high density and/or high temperature of the medium can modify the properties of short-lived resonances such as their masses, widths, and even their spectral shapes [1]. In the final hadronic stage of the collision, due to short lifetime, regeneration and rescattering effects become important and can be used to estimate the timescale between chemical and kinetic freeze-out [2]. Results for $\mathrm{K}^{*}(892)^{0}(c \tau \sim 4 \mathrm{fm})$ and $\phi(1020)(c \tau \sim 45 \mathrm{fm})$ short-lived resonances measured by ALICE in $\mathrm{pp}, \mathrm{p}-\mathrm{Pb}$ and $\mathrm{Pb}-\mathrm{Pb}$ collisions are presented at this seminar.

\section{The ALICE detector}

The ALICE detector [3] [4] at the LHC is designed to study both $\mathrm{Pb}-\mathrm{Pb}$ and pp collisions at the $\mathrm{TeV}$-scale center of mass energy. The components of the ALICE detector most directly related to the results presented here are shortly described below. The Inner Tracking System (ITS) is a silicon detector that surrounds the interaction point and covers the pseudorapidity region $|\eta|<0$.9. It is used to reconstruct the collision vertex and provides tracking and particle identification. Particle tracking is mainly provided by Time Projection Chamber (TPC). The TPC also allows particles to be identified through their energy loss. The Time-of-Flight Detector (TOF) sits after the TPC and measures the time-of-flight of the particles, allowing for additional identification. Both the TPC and the TOF cover the central pseudorapidity region $|\eta|<0.9$. A pair of scintillation hodoscopes, covering $2.8<\eta<5.1$ (VZERO-A) and $-3.7<\eta<-1.7$ (VZERO-B), were used for event triggering and event selection (centrality, multiplicity) in $\mathrm{Pb}-\mathrm{Pb}$ and $\mathrm{p}-\mathrm{Pb}$ collisions.

\section{Analysis Procedure}

The resonances have been reconstructed via their main hadronic decay channel: $\mathrm{K}^{*}(892)^{0} \rightarrow$ $\pi^{ \pm}+\mathrm{K}^{\mp}, \phi(1020) \rightarrow \mathrm{K}^{+}+\mathrm{K}^{-}$. Due to their very short lifetime, resonance decay products cannot be distinguished from the particles coming from the primary vertex, and their yield can only be measured by first computing the invariant mass spectrum of all primary candidate's track pairs and then subtracting the combinatorial background. The combinatorial background was evaluated using the event-mixing technique or the like-sign technique. The signal after the combinatorial background subtraction was then fitted with a Breit-Wigner function for $\mathrm{K}^{* 0}$ or a Voigtian function (convolution of Breit-Wigner and Gaussian which accounts for the detector resolution) for $\phi$ plus a polynomial for the residual background ${ }^{1}$. After the signal is extracted, the raw yield is evaluated as the integral of the signal function. The raw yields extracted in different $p_{\mathrm{T}}$ bins are corrected for efficiency and acceptance and the differential transverse momentum spectra are obtained. The corrected spectra are fitted with a Levy-Tsallis function [5] ( $\mathrm{pp}$ and $\mathrm{p}-\mathrm{Pb}$ collisions) or with a Blast Wave function [6] ( $\mathrm{Pb}-\mathrm{Pb}$ collisions), in order to extract the mean transverse momentum $\left\langle p_{\mathrm{T}}\right\rangle$ and

\footnotetext{
${ }^{1}$ We denote by $\mathrm{K}^{* 0}$ the average of $\mathrm{K}^{*}(892)^{0}$ and $\overline{\mathrm{K}}^{*}(892)^{0}$ and by $\phi$ the $\phi(1020)$ meson
} 
$p_{\mathrm{T}}$-integrated yields. These quantities have been measured for pp collisions at $\sqrt{s}=0.9$ [7], 2.76 and $7 \mathrm{TeV}$ [8], $\mathrm{p}-\mathrm{Pb}$ collisions at $\sqrt{s_{\mathrm{NN}}}=5.02 \mathrm{TeV}$ and $\mathrm{Pb}-\mathrm{Pb}$ collisions at $\sqrt{s_{\mathrm{NN}}}=2.76 \mathrm{TeV}$ [9].

\section{Results}

Examples of the invariant mass spectra for $\mathrm{Pb}-\mathrm{Pb}[9]$ and $\mathrm{p}-\mathrm{Pb}$ collisions are presented in Fig. 1. Masses and widths of resonances are close to the PDG values [10], as illustrated in Fig. 2 for
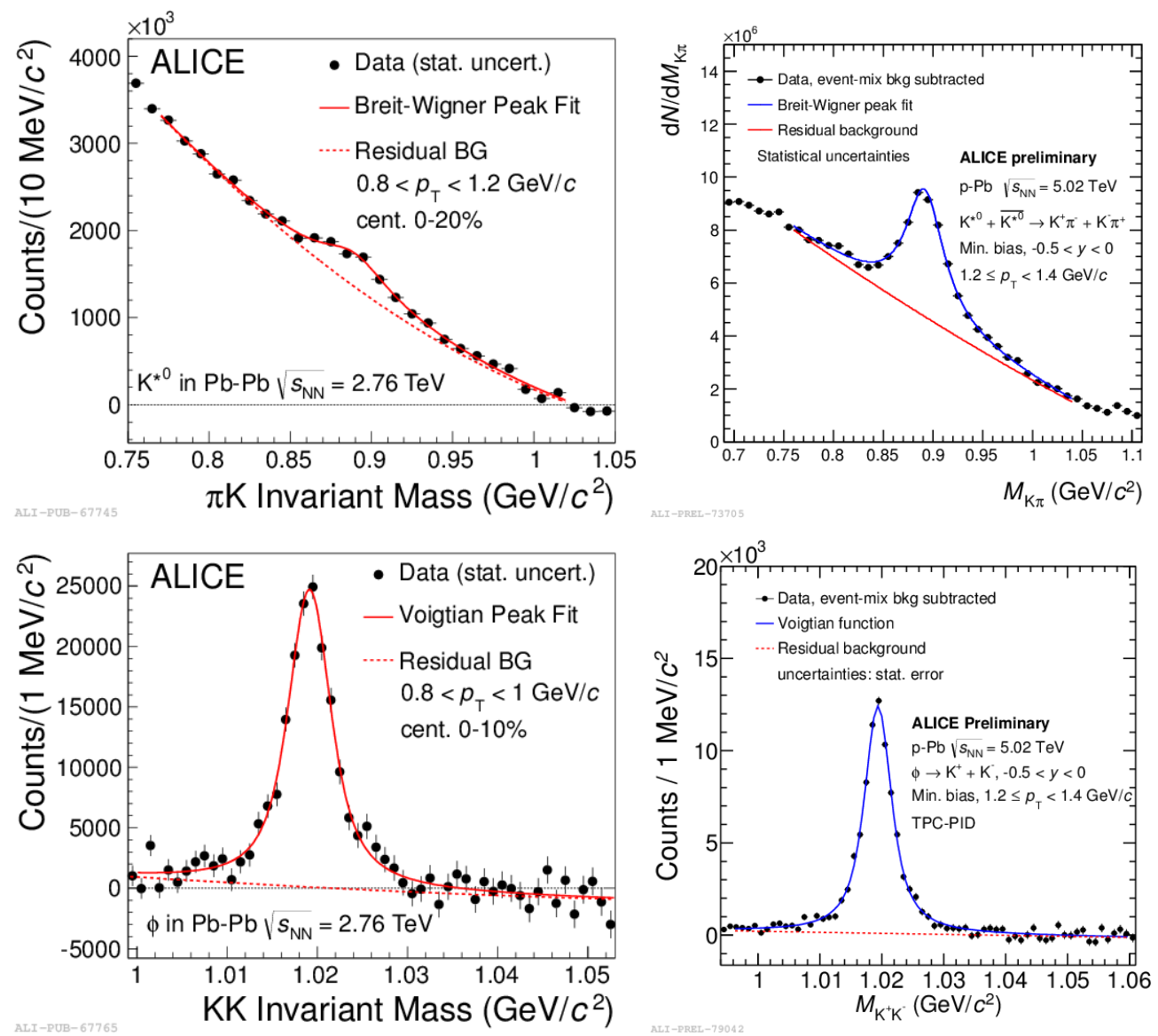

Figure 1: (color online) Signals after the combinatorial background subtraction for $\mathrm{Pb}-\mathrm{Pb}$ (left) [9] and $\mathrm{p}-\mathrm{Pb}$ (right) collisions. The fitting function is the sum of a Breit-Wigner function for $\mathrm{K}^{* 0}$ (top) or a Voigtian function for $\phi$ (bottom) and a polynomial.

$\mathrm{Pb}-\mathrm{Pb}$ collisions [9]. $\mathrm{Pb}-\mathrm{Pb}$ data obtained in 2011 let us to extend measured $p_{\mathrm{T}}$ region of spectra published in [9] up to 10 and $21 \mathrm{GeV} / c$ for $\mathrm{K}^{* 0}$ and $\phi$ respectively, as shown in Fig. 3. Figure 4 shows $\mathrm{K}^{* 0}$ and $\phi$ spectra in $\mathrm{p}-\mathrm{Pb}$ collisions at $\sqrt{s_{\mathrm{NN}}}=5.02 \mathrm{TeV}$ in different event multiplicity classes.

Figure 5 shows $\left\langle p_{\mathrm{T}}\right\rangle$ for the $\mathrm{K}^{* 0}$ and $\phi$ resonances as a function of the mean number of participating nucleons $\left\langle N_{\text {part }}\right\rangle$. For central collisions $\left\langle p_{\mathrm{T}}\right\rangle$ of the $\mathrm{K}^{* 0}(\phi)$ resonance measured in $\mathrm{Pb}-\mathrm{Pb}$ collisions at $\sqrt{s_{\mathrm{NN}}}=2.76 \mathrm{TeV}$ is about $20 \%(30 \%)$ higher than the values measured in $\mathrm{Au}-\mathrm{Au}$ 


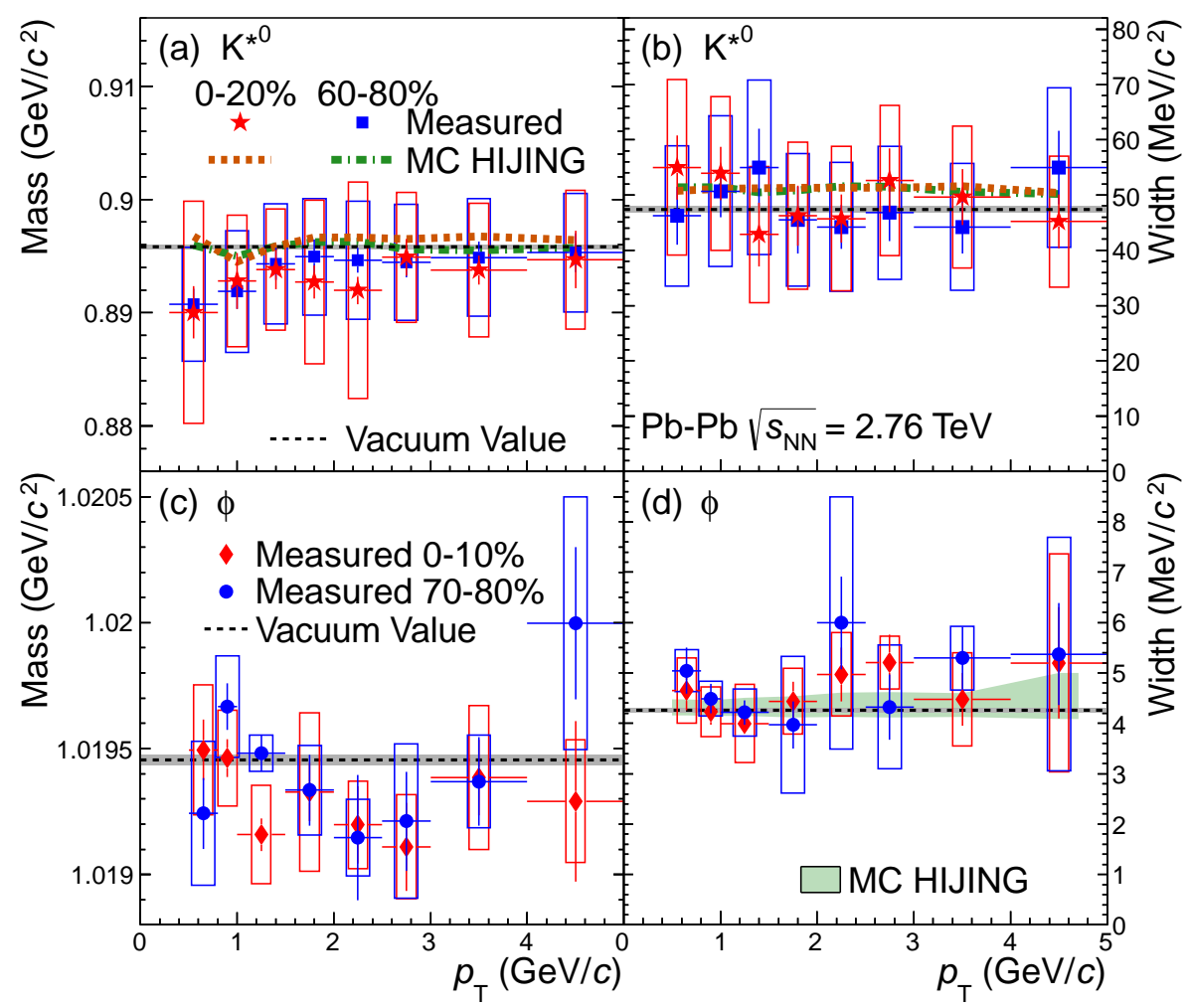

Figure 2: (color online) Mass (left) and width (right) of the $\mathrm{K}^{* 0}$ (top) and $\phi$ (bottom) in $\mathrm{Pb}-\mathrm{Pb}$ collisions at $\sqrt{s_{\mathrm{NN}}}=2.76 \mathrm{TeV}$ as a function of transverse momentum [9].
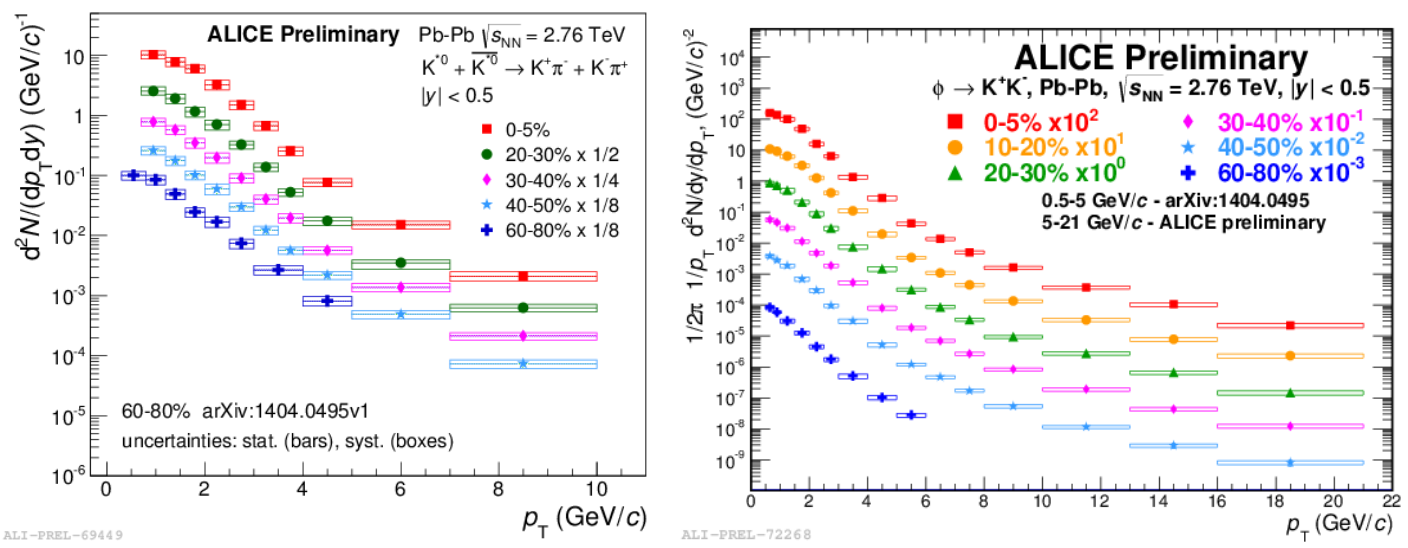

Figure 3: (color online) $\mathrm{K}^{* 0}$ (left) and $\phi$ (right) invariant transverse momentum spectra in $\mathrm{Pb}-\mathrm{Pb}$ collisions at $\sqrt{s_{\mathrm{NN}}}=2.76 \mathrm{TeV}$ in different centrality intervals. 

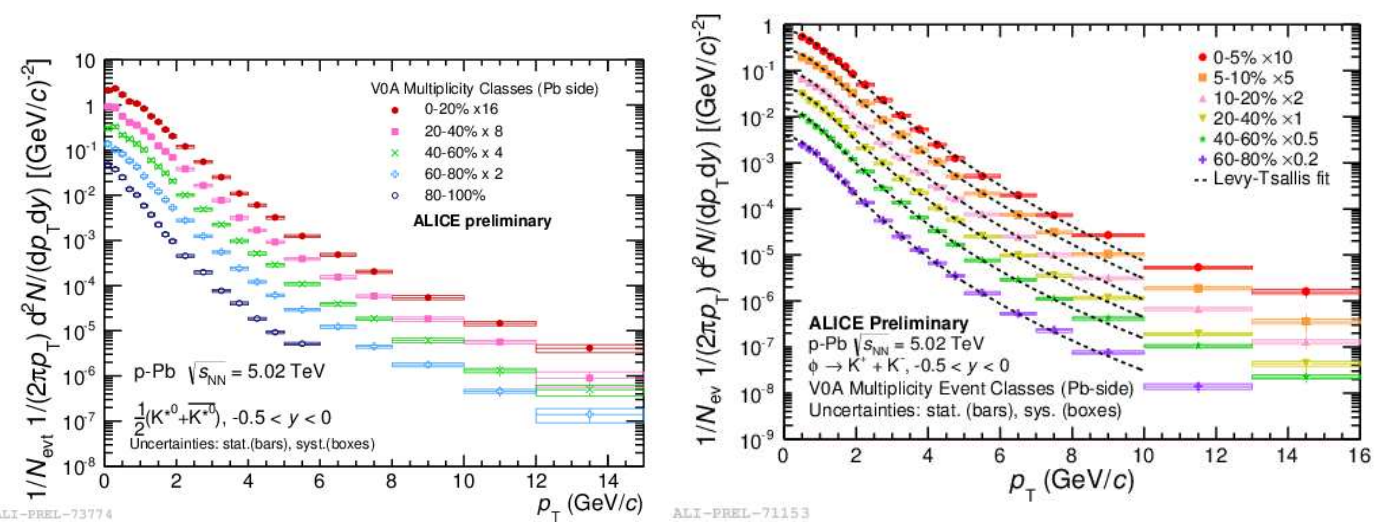

Figure 4: (color online) $\mathrm{K}^{* 0}$ (left) and $\phi$ (right) invariant transverse momentum spectra in $\mathrm{p}-\mathrm{Pb}$ collisions at $\sqrt{s_{\mathrm{NN}}}=5.02 \mathrm{TeV}$ in different event multiplicity classes.

collisions at $\sqrt{s_{\mathrm{NN}}}=200 \mathrm{GeV}$. This is consistent with the observation [11] of increased radial flow in A-A collisions at the LHC relative to RHIC.
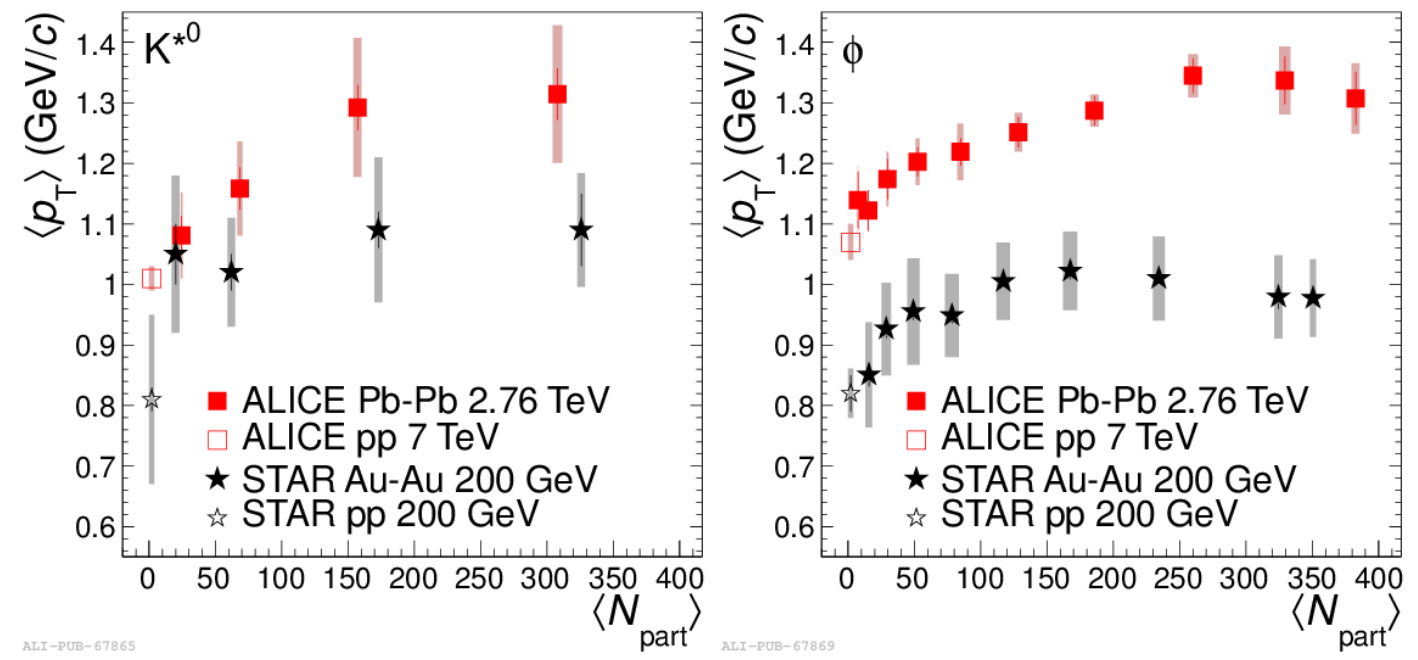

Figure 5: (color online) Mean transverse momentum of the $\mathrm{K}^{* 0}$ (left) and $\phi$ (right) resonances as a function of the mean number of participating nucleons $\left\langle N_{\mathrm{part}}\right\rangle$ in $\mathrm{Pb}-\mathrm{Pb}$ collisions at $\sqrt{s_{\mathrm{NN}}}=2.76 \mathrm{TeV}$ [9]. The results are compared with the pp measurements at $\sqrt{s}=7 \mathrm{TeV}$ and also with the lower energy measurements at RHIC.

In $\mathrm{Pb}-\mathrm{Pb}$ collisions the $\left\langle p_{\mathrm{T}}\right\rangle$ values of the $\mathrm{K}^{* 0}$ and $\phi$ resonances demonstrate similar increasing trend with multiplicity as other hadrons, as shown in Fig. 6. Moreover, mass ordering is observed in central $\mathrm{Pb}-\mathrm{Pb}$ collisions. The $\mathrm{K}^{* 0}, \mathrm{p}$ and $\phi$, which have similar masses, are observed to have similar $\left\langle p_{\mathrm{T}}\right\rangle$ values. This is consistent with hydrodynamic picture. Also in $\mathrm{p}-\mathrm{Pb}$ collisions the $\left\langle p_{\mathrm{T}}\right\rangle$ of resonances increases as a function of the average charged particle multiplicity density, as for other hadrons, Fig. 7. However, while $\left\langle p_{\mathrm{T}}\right\rangle$ of stable hadrons approximately follows mass ordering, the $\left\langle p_{\mathrm{T}}\right\rangle$ of $\mathrm{K}^{* 0}$ and $\phi$ is found to be larger than of protons. A similar trend is observed 

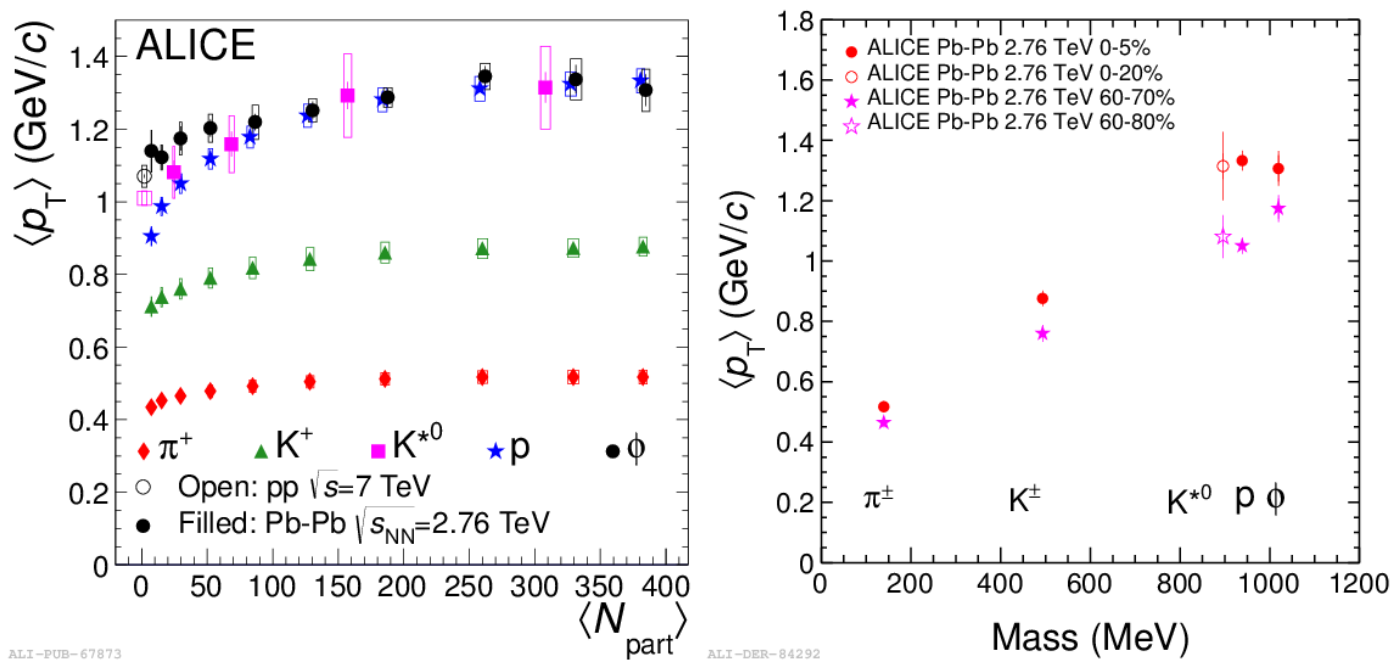

Figure 6: (color online) Mean transverse momentum of the $\mathrm{K}^{* 0}, \phi$ mesons and stable hadrons as a function of the mean number of participating nucleons $\left\langle N_{\text {part }}\right\rangle$ (left) [9] and the particle mass (right) in $\mathrm{Pb}-\mathrm{Pb}$ collisions at $\sqrt{s_{\mathrm{NN}}}=2.76 \mathrm{TeV}$.

in pp collisions at $\left.7 \mathrm{TeV}:\left\langle p_{\mathrm{T}}\right\rangle_{\phi}>\left\langle p_{\mathrm{T}}\right\rangle_{\mathrm{K}^{* 0}}\right\rangle\left\langle p_{\mathrm{T}}\right\rangle_{p}$. The question is open whether the mesonic resonances deviate from mass ordering or the baryons do instead.
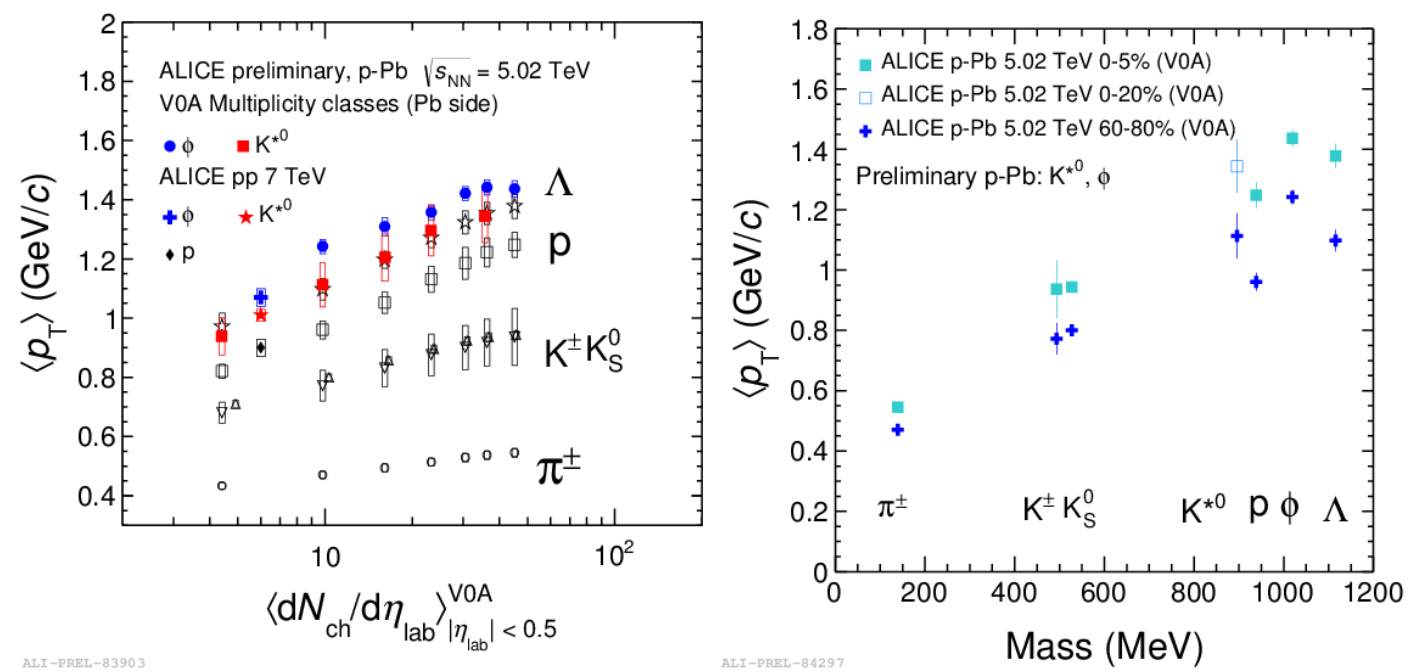

Figure 7: (color online) Mean transverse momentum of the $\mathrm{K}^{* 0}, \phi$ mesons and stable hadrons as a function of the average charged particle multiplicity density (left) and the particle mass (right) in $\mathrm{p}-\mathrm{Pb}$ collisions at $\sqrt{s_{\mathrm{NN}}}=5.02 \mathrm{TeV}$.

Figure 8 shows the $\left\langle p_{\mathrm{T}}\right\rangle$ of $\mathrm{K}^{* 0}$, $\mathrm{p}$ and $\phi$ as a function of the system size in the three collision systems. The $\left\langle p_{\mathrm{T}}\right\rangle$ of resonances in $\mathrm{p}-\mathrm{Pb}$ shows a steeper increase with multiplicity than in $\mathrm{Pb}-\mathrm{Pb}$, as also observed for stable hadrons [12].

Figure 9 (left) presents the particle ratios $\mathrm{K}^{* 0} / \mathrm{K}^{-}$and $\phi / \mathrm{K}^{-}$as a function of the system size 


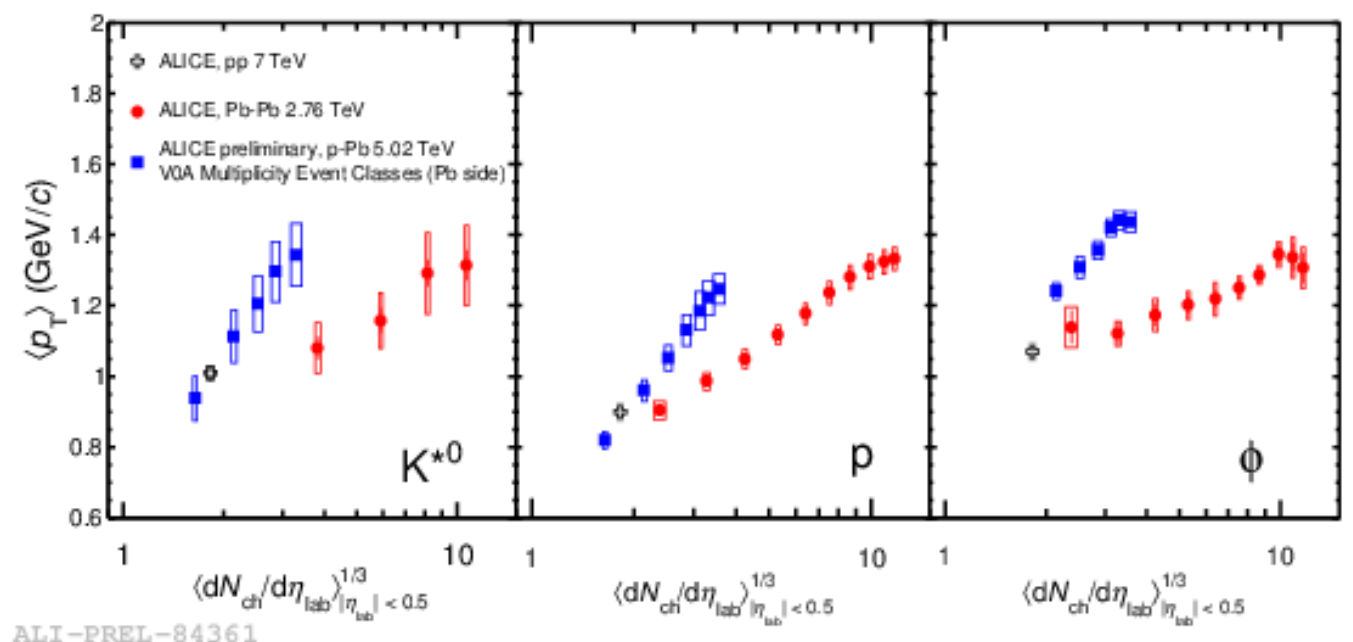

Figure 8: (color online) System size dependence of the mean transverse momentum of the $\mathrm{K}^{* 0}, \mathrm{p}$ and $\phi$. The system size is defined as the cubic root of the average charged particle multiplicity density.

in $\mathrm{pp}, \mathrm{p}-\mathrm{Pb}$ and $\mathrm{Pb}-\mathrm{Pb}$ collisions. The $\mathrm{K}^{* 0} / \mathrm{K}^{-}$ratio exhibits a decreasing trend towards more
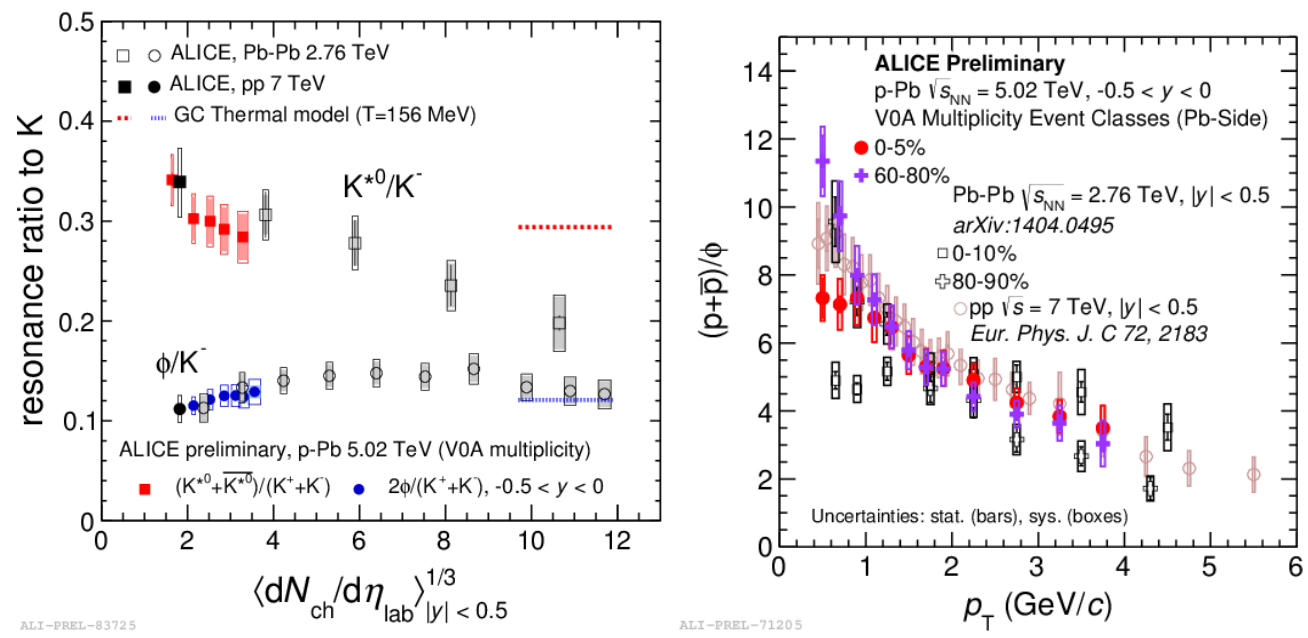

Figure 9: (color online) (left) Ratio of resonances to charged kaons as a function of the system size in the three collision systems [9]. (right) $(\mathrm{p}+\overline{\mathrm{p}}) / \phi$ ratio as a function of transverse momentum in the three collision systems.

central $\mathrm{Pb}-\mathrm{Pb}$ collisions, where the measured ratio is about $60 \%$ of the thermal model prediction with $\mathrm{T}=156 \mathrm{MeV}$ [13]. This suppression is consistent with rescattering of $\mathrm{K}^{* 0}$ daughters in the hadronic phase of central collisions as the dominant effect. As described in [9], the $\mathrm{K}^{* 0} / \mathrm{K}^{-}$ratio measured in central $\mathrm{Pb}-\mathrm{Pb}$ collisions can be used along with the thermal model with rescattering effect [2] to obtain an estimate of $2 \mathrm{fm} / \mathrm{c}$ for the lower limit of the time between chemical and kinetic freeze-out. In $\mathrm{p}-\mathrm{Pb}$ collisions, the $\mathrm{K}^{* 0} / \mathrm{K}^{-}$ratio sits along the extrapolation from pp to 
peripheral $\mathrm{Pb}-\mathrm{Pb}$ collisions. The $\phi / \mathrm{K}^{-}$ratio is nearly flat and agrees with the prediction of the thermal model. This suggests that rescattering effects are not important for $\phi$, which has 10 times longer lifetime than $\mathrm{K}^{* 0}$.

Figure 9 (right) shows the $(\mathrm{p}+\overline{\mathrm{p}}) / \phi$ ratio as a function of transverse momentum in $\mathrm{pp}, \mathrm{p}-\mathrm{Pb}$ and $\mathrm{Pb}-\mathrm{Pb}$ collisions. For central $\mathrm{Pb}-\mathrm{Pb}$ collisions the ratio is flat for $p_{\mathrm{T}}<3-4 \mathrm{GeV} / c$. This is consistent with hydrodynamic description: the shape of a spectrum is determined by the particle mass. The ratio in peripheral $\mathrm{Pb}-\mathrm{Pb}$ collisions decreases steeply with $p_{\mathrm{T}}$. For peripheral $\mathrm{p}-\mathrm{Pb}$ and $\mathrm{pp}$ collisions the ratio is quantitatively consistent, with a trend similar to peripheral $\mathrm{Pb}-\mathrm{Pb}$ collisions. For central $\mathrm{p}-\mathrm{Pb}$ collisions the ratio shows a possible flattening for $p_{\mathrm{T}}<1.5 \mathrm{GeV} / c$, which could be interpreted as a hint for the onset of collective behavior in these collisions.

Figure 10 (left) shows the nuclear modification factors of the $\mathrm{K}^{* 0}$ and $\phi$ resonances and stable hadrons as a function of $p_{\mathrm{T}}$ for central $\mathrm{Pb}-\mathrm{Pb}$ collisions at $\sqrt{s_{\mathrm{NN}}}=2.76 \mathrm{TeV}$. At high $p_{\mathrm{T}}$ both
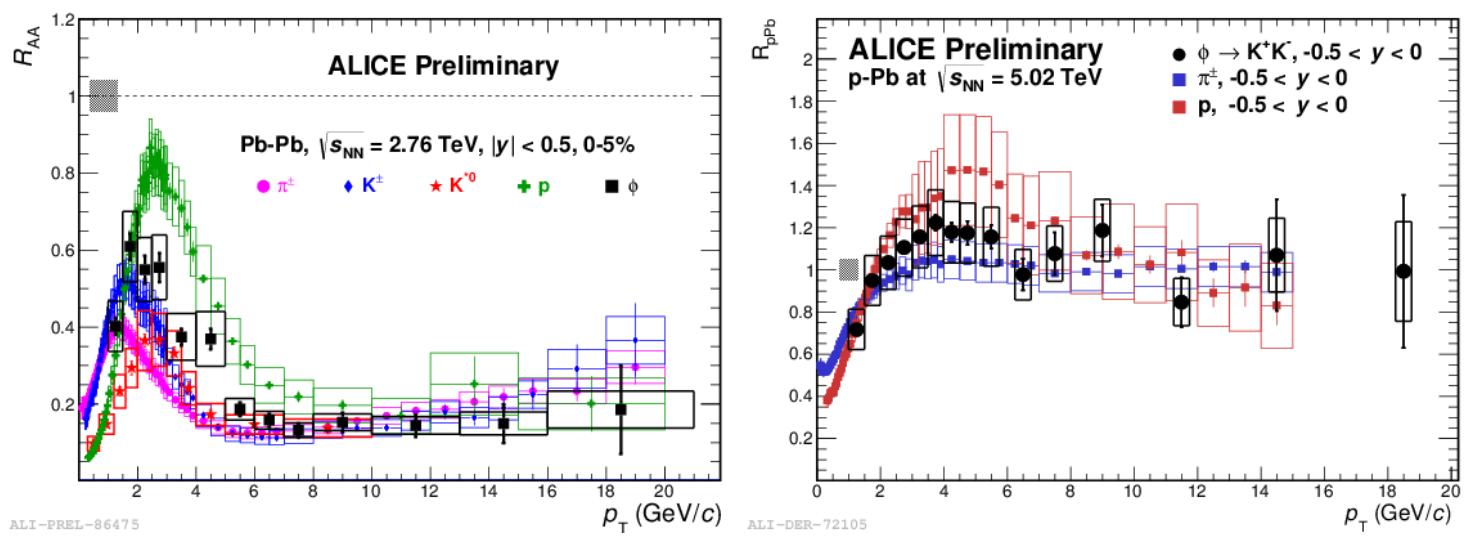

Figure 10: (color online) Nuclear modification factor of resonances and stable hadrons as a function of transverse momentum for central $\mathrm{Pb}-\mathrm{Pb}$ collisions at $\sqrt{s_{\mathrm{NN}}}=2.76 \mathrm{TeV}$ (left) and for minimum bias $\mathrm{p}-\mathrm{Pb}$ collisions at $\sqrt{s_{\mathrm{NN}}}=5.02 \mathrm{TeV}$ (right).

$\mathrm{K}^{* 0}$ and $\phi$ strongly suppressed with respect to pp collisions, $R_{A A} \approx 0.15-0.2$. The suppression is consistent with that measured for the stable hadrons, supporting the flavor-independence of partonic energy loss in the medium. At intermediate $p_{\mathrm{T}}, 2<p_{\mathrm{T}}<6 \mathrm{GeV} / c, R_{A A}\left(\pi^{ \pm}, \mathrm{K}^{ \pm}, \mathrm{K}^{* 0}\right)<R_{A A}(\phi)<$ $R_{A A}(\mathrm{p})$. At low $p_{\mathrm{T}}, p_{\mathrm{T}}<2 \mathrm{GeV} / c$, the $\mathrm{K}^{* 0}$ is more suppressed than charged hadrons, which may be an indication that rescattering effects are important. Figure 10 (right) shows the nuclear modification factors of the $\phi$ meson and stable hadrons in minimum bias $\mathrm{p}-\mathrm{Pb}$ collisions at $\sqrt{s_{\mathrm{NN}}}$ $=5.02 \mathrm{TeV}$. There is no suppression at high $p_{\mathrm{T}}, R_{p P b}$ does not deviate from unity for $\pi^{ \pm}, \mathrm{p}$ and $\phi$. At intermediate $p_{\mathrm{T}}$ there is a moderate Cronin peak.

\section{Conclusions}

The $\mathrm{K}^{* 0}$ and $\phi$ resonance production have been measured in a wide momentum range in $\mathrm{pp}$, $\mathrm{p}-\mathrm{Pb}$ and $\mathrm{Pb}-\mathrm{Pb}$ collisions by the ALICE experiment at the LHC. The masses and widths of the resonances are close to the PDG values. In $\mathrm{pp}$ and $\mathrm{p}-\mathrm{Pb}$ collisions the resonance $\left\langle p_{\mathrm{T}}\right\rangle$ does not follow the same mass ordering as in central $\mathrm{Pb}-\mathrm{Pb}$ collisions. The $\mathrm{K}^{* 0} / \mathrm{K}^{-}$ratio exhibits a significant 
suppression going from peripheral to most central $\mathrm{Pb}-\mathrm{Pb}$ collisions, consistent with rescattering of $\mathrm{K}^{* 0}$ decay daughters in the hadronic phase due to short lifetime of $\mathrm{K}^{* 0}$. The $\phi / \mathrm{K}^{-}$ratio is not suppressed due to its longer lifetime. In central $\mathrm{Pb}-\mathrm{Pb}$ collisions the $(\mathrm{p}+\overline{\mathrm{p}}) / \phi$ ratio is flat for $p_{\mathrm{T}}<3-4 \mathrm{GeV} / c$, which is consistent with hydrodynamic description. For central $\mathrm{p}-\mathrm{Pb}$ collisions the ratio demonstrates a possible flattening for $p_{\mathrm{T}}<1.5 \mathrm{GeV} / c$, which could be interpreted as a hint for the onset of collective behavior in these collisions. In central $\mathrm{Pb}-\mathrm{Pb}$ collisions the nuclear modification factor $R_{\mathrm{AA}}$ of $\mathrm{K}^{* 0}$ and $\phi$ resonances are strongly suppressed at high- $p_{\mathrm{T}}$, consistent with the suppression for stable hadrons. There is no suppression at high $p_{\mathrm{T}}$ in minimum bias $\mathrm{p}-\mathrm{Pb}$ collisions, $R_{\mathrm{pPb}}$ does not deviate from unity for $\pi^{ \pm}$, $\mathrm{p}$ and $\phi$.

\section{References}

[1] G. E. Brown and M. Rho, Phys. Rev. Lett. 66, (1991) 2720. R. Rapp, Nucl. Phys. A 725, (2003) 254. E. V. Shuryak and G. Brown, Nucl. Phys. A 717, (2003) 322.

[2] G. Torrieri and J. Rafelski, Phys. Lett. B 509, (2001) 239. J. Rafelski, J. Letessier and G. Torrieri, Phys. Rev. C 64 (1993) 054907. C. Markert, J. Rafelski and G. Torrieri, arXiv:0206260 [hep-ph].

[3] K. Aamodt et al., (ALICE Collaboration), JINST 3, (2008) S08002.

[4] B. Abelev et al., (ALICE Collaboration), Int. J. Mod. Phys. A 29 (2014) 1430044.

[5] C. Tsallis, J. Stat. Phys. 52, (1988) 479.

[6] E. Schnedermann, J. Sollfrank and U. Heinz, Phys. Rev. C 48 (1993) 2462

[7] K. Aamodt et al., (ALICE Collaboration), Eur. Phys. J. C 71 (2011) 1594.

[8] K. Aamodt et al., (ALICE Collaboration), Eur. Phys. J. C 72 (2012) 2183.

[9] B. Abelev et al., (ALICE Collaboration), arXiv:1404.0495 [hep-ex].

[10] J. Beringer et al., (Particle Data Group), Phys. Rev. D 86, (2012) 010001.

[11] B. Abelev et al., (ALICE Collaboration), Phys. Rev. C 88, (2013) 044910.

[12] B. Abelev et al., (ALICE Collaboration), Phys. Lett. B 727, (2013) 371.

[13] J. Stachel et al., J. Phys.: Conf. Ser. 509, (2014) 012019. 